\begin{tabular}{|c|c|c|}
\hline & This Journal is available in Telkom University online Journals & $::::::::::{ }^{\prime}$ \\
\hline & Jurnal Manajemen Indonesia & ::::::::::::: \\
\hline Telkom & Journal homepage: journals.telkomuniversity.ac.id/ijm & $\begin{array}{l}\text { JURNAL } \\
\text { MANAJEMEN } \\
\text { INDONESIA }\end{array}$ \\
\hline
\end{tabular}

\title{
Customer Perception on Prulink Product Purchase Decision: A Case of Greater Jakarta Area
}

\author{
Rendika Nugraha ${ }^{1}$, Farida Komalasari ${ }^{2}$, Sandra Dethionia ${ }^{3}$ \\ ${ }^{1}$ President University, Bekasi, Indonesia \\ ${ }^{2}$ President University, Bekasi, Indonesia \\ ${ }^{3}$ President University, Bekasi, Indonesia
}

\begin{abstract}
Purpose of this research is to find out the influence of customer perception of unit link insurance product (Prulink) purchase decision through four aspects which are; information, premium, investment and risk. This research used quantitative analysis approach utilizing questionnaire, which consist of 20 items of questions as the instrument. Questionnaires were spread to customer of PT. Prudential Life Assurance Jakarta that located in Jakarta greater area as population sampling of this research. Data analysis was conducted by checking the validity (KMO and Bartlett't Test) and reliability (Cronbach Alpha) of the data. Inferential analysis was conducted using multiple regression analysis to check relationship between dependent variable and independent variable. Pre-test has been conducted to 30 people with results that questionnaire reliable to be distributed after obtain .974 on Cronbach's Alpha, validity in range .751 to .933 and reliability in range of .858 to .936 of 200 samples data that shows that each dependent and independent variables passed to hypothesis testing with multiple regression analysis. Towards this research, it is found that three aspects of customer perception, which are information, premium, and investment, have significant influence on purchase decision. On the other hand, there is no significant partial influence from risk aspect. Combining the common variable (Information, premium, and risk) with specific variable (investment) believed to become uniqueness of this research considering there might be other studies which have similar variable but without combining common variable with specific variable.
\end{abstract}

Keywords-Customer Perception; Information; Premium; Investment; Purchase decision; Insurance.

\begin{abstract}
Abstrak
Tujuan dari penelitian ini untuk mengetahui pengaruh dari persepsi pelanggan dari produk asuransi unit link (Prulink) melalui empat aspek keputusan pembelian yaitu informasi, premi, investasi dan resiko. Penelitian ini menggunakan metode kuantitatif dengan kuosioner yang terdiri dari dua puluh pertanyaan sebagai instrument. Kuosioner disebarkan kepada pelangan dari PT. Priudential life Assurance Jakarta yang terletak di area Jakarta sebagai sample populasi dari penelitian ini. Analisa data dilakukan dengan menggunakan tes validitas (KMO and Bartlett't Test) dan reliabilitas (Cronbach Alpha). Analisa inferensial dilakukan dengan analisis regresi berganda untuk mengetahui hubungan antara variabel tak bebas dan variabel bebas. Ujicoba telah dilakukan kepada tiga puluh orang dengan hasil bahwa kuosioner layak untuk disebarkan setelah mendapatkan nilai .974 pada Cronbach's alpha, validitas dalam cakupan .751 sampai .933 dan reliabilitas dalam cakupan .858 sampai .936 sampai dari 200 data sampel yang menunjukkan bahwa variabel tak bebas dan variabel bebas telah lulu uji hipotesis dengan analisis regresi berganda. Melalui penelitian ini, ditemukan tiga aspek dari persepsi pelanggan yaitu informasi, premi dan investasi yang mempunyai pengaruh yang signifikan terhadap keputusan pembelian. Di sisi lain, tidak ada pengaruh parsial yang signifikan dari aspek resiko. Mengombinasikan variabel umum (informasi, premi, dan resiko dengan variabel khusus (investasi) dipercaya menjadi keunikan dari riset ini mengingat ada kemungkinan dari penelitian/studi lain yang mempunyai variabel yang sama tetapi tanpa mengombinasikan variabel umum dengan variabel khusus.
\end{abstract}

Kata kunci- Persepsi Pelanggan; Informasi; Premi; Investasi; Keputusan Pembelian; Asuransi.

\section{Article info}

Received ( $1^{\text {st }}$ August, 2018)

Revised (16 ${ }^{\text {th }}$ August, 2018)

Accepted (25 $5^{\text {th }}$ August, 2018)

Corresponding_author: rendika@president.ac.id 


\section{INTRODUCTION}

Insurance and investment have already common terms in Indonesia especially for people who work and live in the big cities especially in Jakarta greater area. Having insurance with purpose to protect their life from financial risk that caused by unwanted situation in life such as accident, death, disaster, and etc. According to Huitt (2007), being secure or safe is the second level of human needs. In that case, insurance has become an option to mitigate the risk. Many insurance company in Indonesia have similar message in their advertisement is to give sense of security and assurance in the future. According to Law no. 2/1992 about insurance business in Indonesia, Many insurance companies found that Indonesia as a good target market to develop insurance product. In 2016, the number of insurance income increased up to 78.1\% (Bisnis.com, 2016). Meanwhile, partial withdrawal of insurance product declined as $40.4 \%$ (Beritasatu.com, 2016).

Nowadays, there are two insurance types for individual customer, traditional insurance and unit link insurance. Traditional insurance has function to protect, meanwhile unit link insurance has two functions protect, and investment. Unit link insurance becomes an option for people who want to be protected as well as have investment at the same time. Premium in unit link product divided into insurance charge and also unit of investment. In 2015, Unit link insurance in Indonesia supplied 72 trillion rupiah, whereas traditional insurance supplied 56 trillion rupiah, meaning that $60 \%$ of total premium income of insurance in Indonesia came from unit link product (Infobanknews.com, 2016).

Prudential is the first insurance company that offered unit link insurance in Indonesia since 1999 with name Prulink. Prudential also serve a market leader for unit link insurance product in Indonesia. S one of the unit link insurance provider in Indonesia, Prudential should understand the factor that generate customer purchase decision to buy the unit link product because satisfied customer will result loyalty and customer retention. In that case, provider should be more innovative and think of new ways in approaching potential customer to meet customer expectation and perception (Rajeswari \& Kartheeswari, 2011).

Perception created from the experience, expectation, and also interest. In case of unit link insurance product, customers usually need to collect clear information about the product and premium in order to consider their life risk and calculate the benefit from unit link investment before purchase the unit link insurance product. Based on that, researcher, consider several aspect of perception that influences customer purchase decision in purchasing unit link insurance product. Analysis focuses on information, premium, investment, and also risk. This research were done in Jakarta greater area to customers of Prudential unit link (Prulink) insurance product, where Jakarta considered as big city in Indonesia and people have sufficient literacy about insurance and investment.

\section{LITERATURE REVIEW}

\section{A. Insurance and Unit Link}

According to Bhatnagar (2015), Insurance is a social device to reduce the risk of life and a shield to face the risks and unforeseen circumstances. Unit link is one of the types of life insurance product; it is a life insurance combine with another investment plan that gives opportunity for customer to invest in several investments (Standard Life, 2016). Bhatnagar (2015) also stated that unit link insurance product is a life insurance solution that provides benefits of protection and flexibility in investment where the investment indicated as units and represented by the value that it has attained called as Net Asset Value.

\section{B. Purchase Decision}

Lovelock \& Wirtz (2007) described purchasing process of experiencing the selecting, taking, and perceive a service. Purchasing process consists of three stages which are; pre-purchase stage, service encounter stage, and post encounter stage. Pre-purchase stage is where customers observes and try to match the needs with service offered in order to make purchase decision In this stage, external factors have the role to help customer meet their needs. In service encounter stage, customer contacts the service provider and there is an interacting to deliver the service. In this stage, there is two kinds of contact service, low and high. In low contact service, customer and service provider usually communicate through website, phone, and email. In high contact service, customer and service provider usually meet face to face in order to purchase the service. Last stage is most encounter stage; customers already experience the service and evaluate their satisfaction towards the service 
based on customer expectation and perception. Evaluation would results in continue to use the service or have no intention to continue using the service in the future.

\section{Customer Perception}

Perception described as individual process where the sensory impressions interpreted and organized to create a meaning in the reality. In this situation, behavior is driven by individual perception. There are three factors that influence customer perception such as; perceiver, target, and situation (Robbins \& Judge, 2011). In this research, exploration focused more in perceiver type. Perception caused by perceiver means that the perception created based on personal expectation, experiences, interest or motives of the individual. Reddy \& Jahangir (2015), on their research stated that perception is one of the factors that affect purchase intention.

\section{Information}

Information is important for insurance in order to raise public awareness of the benefits of insurance. Quality of information itself depends on three things such as; Accuracy of the information not to be false or misleading, Just in time mans the information should not be late to be received by recipient, relevancy means that the information should have benefit to the recipient. Information has positive influence on the purchase intention in the future (Reddy \& Jahangir, 2015). In the service industry itself, Information processing has a big role in selling banking, accounting, and insurance product (Lovelock \& Wirtz, 2007).

\section{E. Premium}

The insurance premium is the obligation of the insurer to the insured party in form of payment of certain amount of money that agreed to be paid periodically. The amount of premium depends on factors that lead to a high risk and low levels of total value instead (Redja, 2011). Ulbinaite, Kucinskiene, \& Moullec (2014) on their study found that affordable premium triggers customer tendency to purchase the insurance product without paying too much attention to the quality or the policy content.

\section{F. Investment}

Bhatnagar (2015), stated that unit link has less risk in comparison to other insurance product. Return from unit link insurance product excluded from the tax, which make people enjoy and satisfy to purchase unit link insurance product. Bhatnagar (2015), also stated that the advantage unit link insurance plan are simple, clear and easy to understand.

\section{G. Risk}

According to Redja (2011), risk is defined as uncertainty concerning the occurrence of a loss. Furthermore, risk can be classified into several distinct classes, which are pure \& speculative risk, diversifiable $\&$ nondiversifiable risk, and enterprise risk. In life insurance, pure risk scheme is applied for customers as pure risk is defined as a situation in which there are only the possibilities of loss (adverse) or no loss (neutral).

\section{H. Research Gaps}

Based on previous research (Reddy \& Jahangir, 2015), found that the most significant influence towards purchase decision is information. Moreover, another study found that the most significant factor among the customer perceptions to get future purchase is premium (Sebjan \& Tomic, 2015). Bhatnagar (2015), has shown that the main factor that led people aware of unit link is investment. Based on some previous research, researcher focused on the influence of information, premium, investment, and risk as customer perception on unit link purchase decision. Some of previous research used service quality, and marketing mix as the variable to test purchase decision, meanwhile in this research, investment based on the unit link insurance product was added as investment instrument. 


\section{Metode Penelitian}

\section{A. Research Gaps}

Quantitative methodology was applied in conducting this research in order to find out the influence of customer perception on Prulink product purchase decision. Based on the objectives of this research, theoretical framework were established and shown in figure 1

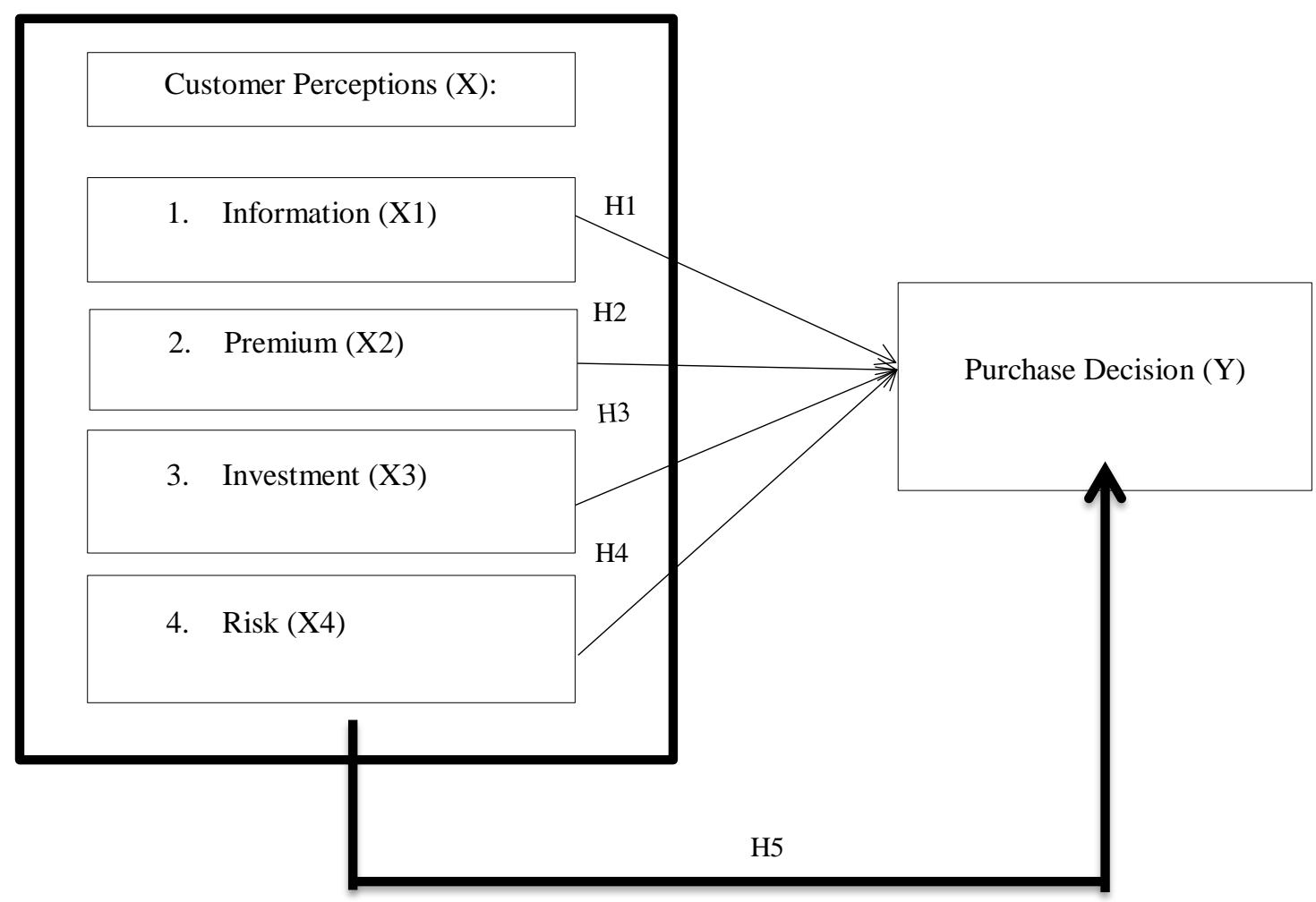

Figure 1 Theoretical Framework

Based on the theoretical framework, there are five hypotheses that have been tested:

H1 : Information influences costumer purchase decision

H2 : Premium influences costumer purchase decision

H3 : Investment influences costumer purchase decision

H4 : Risk influences costumer purchase decision

H5 : All aspects of costumer perception influence purchase decision altogether

\section{B. Operational and Definitions of Variable}

There are two main variables that used in this research, first is customer perception, which divided into four aspects of information, premium, investment, and risk as the indicator. Second, purchase decision. Table 1 explains the operational and definitions of variables as well as the construct of question in the questionnaire based on the variables.

Table 1. Operational and Definitions of Variable

\begin{tabular}{|c|c|c|c|c|}
\hline Variable & Definition/Indicator & Original Question & Adjusted Question & Sources \\
\hline $\begin{array}{l}\text { Customer } \\
\text { Perception }\end{array}$ & Information & $\begin{array}{l}\text { The insurance company } \\
\text { provides me with } \\
\text { detailed information } \\
\text { about the insurance }\end{array}$ & $\begin{array}{l}\text { Prudential provides me } \\
\text { with detailed } \\
\text { information about the } \\
\text { Unit Link product }\end{array}$ & $\begin{array}{l}\text { Sebjan, U. } \\
\text { and Tominc, } \\
\text { P. (2015) }\end{array}$ \\
\hline
\end{tabular}




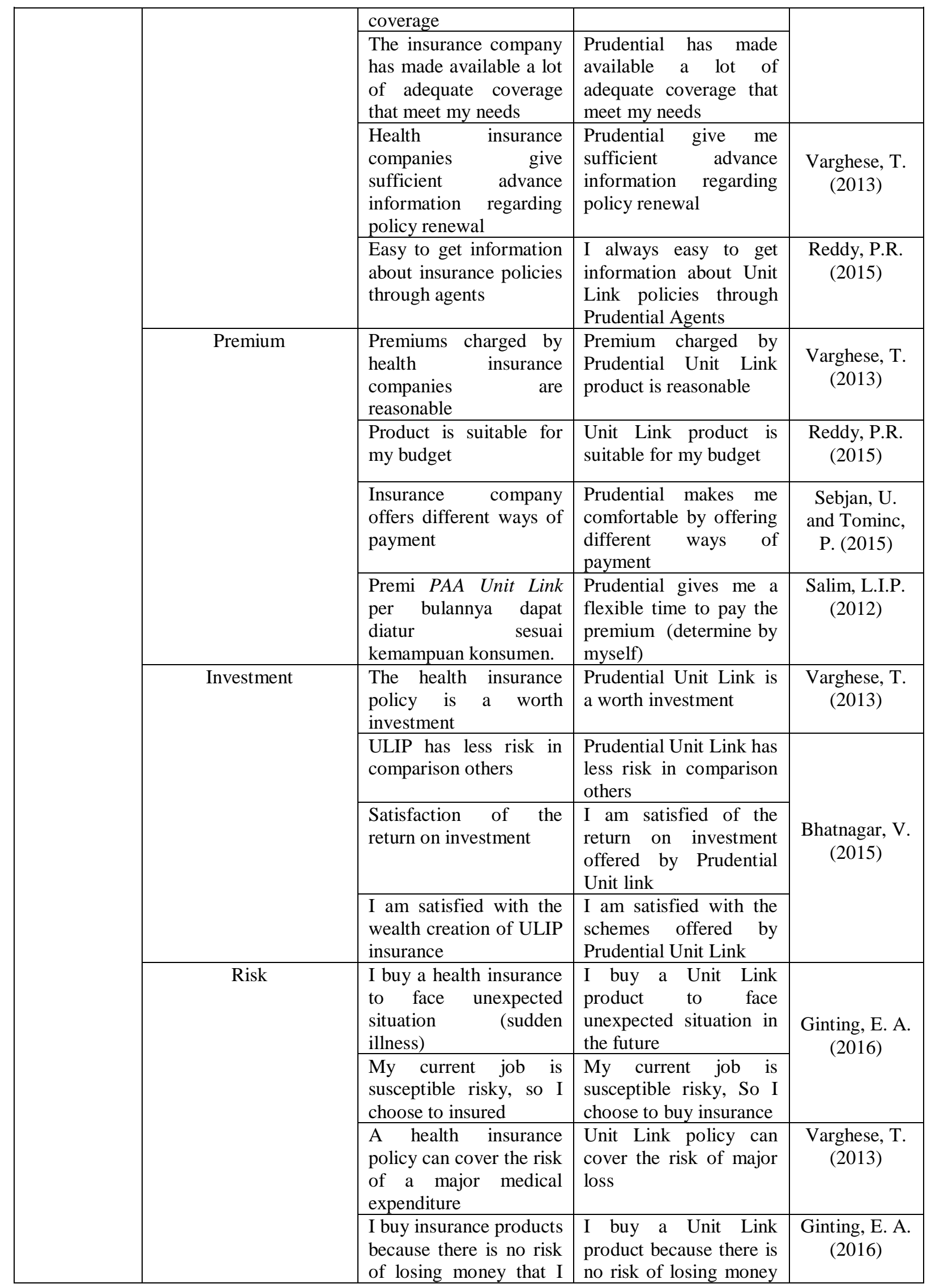




\begin{tabular}{|c|c|c|c|}
\hline & paid & that I paid & \\
\hline \multirow[t]{4}{*}{$\begin{array}{l}\text { Purchase } \\
\text { Decision }\end{array}$} & $\begin{array}{l}\text { Before deciding to } \\
\text { purchase PAA unit link } \\
\text { Product, you think it is } \\
\text { important to find } \\
\text { information regarding } \\
\text { offered package. }\end{array}$ & $\begin{array}{l}\text { Before I decide to } \\
\text { purchase Prudential } \\
\text { Unit Link, I have to } \\
\text { look for product } \\
\text { information actively }\end{array}$ & \multirow{4}{*}{$\begin{array}{c}\text { Bhatnagar, } \mathrm{V} \text {. } \\
(2015)\end{array}$} \\
\hline & $\begin{array}{l}\text { After get the } \\
\text { information regarding } \\
\text { PAA Unit link, it is } \\
\text { important to you to } \\
\text { evaluate and consider } \\
\text { wisely before make a } \\
\text { decision }\end{array}$ & $\begin{array}{l}\text { After I get the } \\
\text { information, I have to } \\
\text { evaluate and consider } \\
\text { carefully before making } \\
\text { decisions }\end{array}$ & \\
\hline & $\begin{array}{l}\text { You consider that your } \\
\text { decision to use PAA } \\
\text { unit link is right } \\
\text { decision. }\end{array}$ & $\begin{array}{l}\text { I think to purchase } \\
\text { Prudential Unit Link is } \\
\text { the right decision }\end{array}$ & \\
\hline & $\begin{array}{l}\text { You feel satisfy with the } \\
\text { benefit that PAA Unit } \\
\text { link offered because it } \\
\text { can fulfill needs of life } \\
\text { and health protection. }\end{array}$ & $\begin{array}{l}\text { I am satisfied with the } \\
\text { benefit offered because } \\
\text { it fulfill the needs of } \\
\text { guarantee life and health }\end{array}$ & \\
\hline
\end{tabular}

Source: Developed by Author

\section{Sampling Design}

Customers of PT. Prudential Life Assurance in Jakarta above 25 years old are sampling population of this research. As for the sample size, researcher utilized ratio 10:1 in order to become accepted sample size. Since the amounts of item were 20, thus the total sample size would be 200 samples. Researcher used non-probability sampling design type quota sampling to ensure the accuracy of sample to be similar with population (Malhotra, 2009).

\section{RESUlt AND DisCUSSION}

\section{A. Validity Test}

Factor analysis was utilized to check the validity, while during testing, dependent and independent variables were checked separately. The result of dependent variable shows Kaiser-Meyer-Olkin (KMO) is .751 and Bartlett's Test have sig. value of (.000). The value Communalities indicate all dependent items exceed that 0.5 ; PRDC1 (.930), PRDC2 (.913), PRDC3 (.940), PRDC4 (.945) and Total Variance Cumulative 93\% means all the items of dependent variable pass the Validity test, before followed by Rotated Component Matrix loads in one factor.

Table 2. KMO and Bartlett's Test - Dependent Variable

\begin{tabular}{|ll}
\hline Kaiser-Meyer-Olkin Measure of Sampling Adequacy. \\
& Approx. Chi-Square \\
Bartlett's Test of Sphericity & df \\
& Sig. \\
\hline
\end{tabular}


Table 3. Communalities - Dependent Variable

\begin{tabular}{|l|r|r|}
\hline & Initial & Extraction \\
\hline PRDC1 & 1.000 & .930 \\
PRDC2 & 1.000 & .913 \\
PRDC3 & 1.000 & .940 \\
PRDC4 & 1.000 & .945 \\
\hline
\end{tabular}

Extraction Method: Principal Component Analysis.

Table 4. Total Variance Explained - Dependent Variable

\begin{tabular}{|l|r|r|r|r|r|r|r|r|r|}
\hline \multirow{2}{*}{ Component } & \multicolumn{4}{|c|}{ Initial Eigenvalues } & \multicolumn{3}{c|}{$\begin{array}{c}\text { Extraction Sums of Squared } \\
\text { Loadings }\end{array}$} & \multicolumn{3}{c|}{$\begin{array}{c}\text { Rotation Sums of Squared } \\
\text { Loadings }\end{array}$} \\
\cline { 2 - 10 } & Total & $\begin{array}{c}\text { \% of } \\
\text { Variance }\end{array}$ & $\begin{array}{c}\text { Cumulative } \\
\%\end{array}$ & Total & $\begin{array}{c}\text { \% of } \\
\text { Variance }\end{array}$ & $\begin{array}{c}\text { Cumulative } \\
\%\end{array}$ & Total & $\begin{array}{c}\text { \% of } \\
\text { Variance }\end{array}$ & $\begin{array}{c}\text { Cumulative } \\
\%\end{array}$ \\
\hline 1 & 3.225 & 80.615 & 80.615 & 3.225 & 80.615 & 80.615 & 1.887 & 47.186 & 47.186 \\
2 & .503 & 12.585 & 93.200 & .503 & 12.585 & 93.200 & 1.841 & 46.014 & 93.200 \\
3 & .159 & 3.966 & 97.165 & & & & & & \\
4 & .113 & 2.835 & 100.000 & & & & & & \\
\hline
\end{tabular}

Extraction Method: Principal Component Analysis.

Table 5. Rotated Component Matrix ${ }^{\mathrm{a}}$ - Dependent Variable

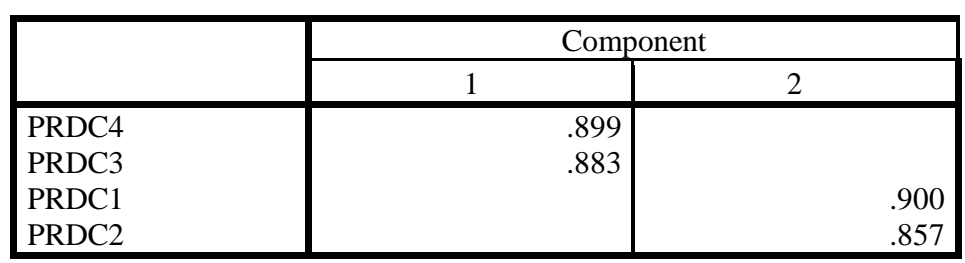

Extraction Method: Principal Component Analysis.

Rotation Method: Varimax with Kaiser Normalization.

a. Rotation converged in 3 iteration

From independent variables, there were 4 questions that need to be deleted due to failing pass the requirements, which are INFO4, INVES1, INVES3, and RISK3. After deletion of the questions that did not pass the requirement, the KMO shows .933 and Bartlett's Test have sig. value (.000). The rest of items also get above 0.5 for Communalities with Total Variance of $86.6 \%$. Each item also loads perfectly in one factor, so all the Independent pass the Validity Test.

Table 6. KMO and Bartlett's Test - Independent Variable

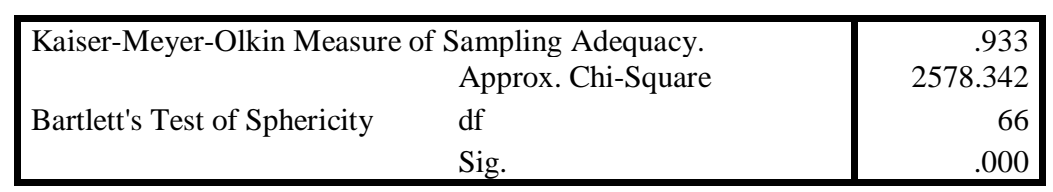


Table 7. Communalities - Independent Variables

\begin{tabular}{|l|r|r|}
\hline & \multicolumn{1}{|c|}{ Initial } & Extraction \\
\hline INFO1 & 1.000 & .885 \\
INFO2 & 1.000 & .786 \\
INFO3 & 1.000 & .902 \\
PREMI1 & 1.000 & .872 \\
PREMI2 & 1.000 & .907 \\
PREMI3 & 1.000 & .847 \\
INVES2 & 1.000 & .901 \\
INVES4 & 1.000 & .877 \\
RISK2 & 1.000 & .881 \\
RISK4 & 1.000 & .834 \\
PREMI4 & 1.000 & .857 \\
RISK1 & 1.000 & .848 \\
\hline
\end{tabular}

Extraction Method: Principal Component Analysis.

Table 8. Total Variance Explained - Independent Variables

\begin{tabular}{|c|c|c|c|c|c|c|c|c|c|}
\hline \multirow[t]{2}{*}{ Component } & \multicolumn{3}{|c|}{ Initial Eigenvalues } & \multicolumn{3}{|c|}{$\begin{array}{c}\text { Extraction Sums of Squared } \\
\text { Loadings }\end{array}$} & \multicolumn{3}{|c|}{$\begin{array}{c}\text { Rotation Sums of Squared } \\
\text { Loadings }\end{array}$} \\
\hline & Total & $\begin{array}{c}\% \text { of } \\
\text { Variance }\end{array}$ & $\begin{array}{c}\text { Cumulative } \\
\%\end{array}$ & Total & $\begin{array}{c}\% \text { of } \\
\text { Variance }\end{array}$ & $\begin{array}{c}\text { Cumulative } \\
\%\end{array}$ & Total & $\begin{array}{c}\% \text { of } \\
\text { Variance }\end{array}$ & $\begin{array}{c}\text { Cumulative } \\
\%\end{array}$ \\
\hline 1 & 8.708 & 72.569 & 72.569 & 8.708 & 72.569 & 72.569 & 3.246 & 27.053 & 27.053 \\
\hline 2 & .726 & 6.051 & 78.620 & .726 & 6.051 & 78.620 & 2.846 & 23.720 & 50.774 \\
\hline 3 & .518 & 4.317 & 82.937 & .518 & 4.317 & 82.937 & 2.289 & 19.076 & 69.850 \\
\hline 4 & .444 & 3.699 & 86.637 & .444 & 3.699 & 86.637 & 2.014 & 16.787 & 86.637 \\
\hline 5 & .354 & 2.949 & 89.586 & & & & & & \\
\hline 6 & .303 & 2.525 & 92.111 & & & & & & \\
\hline 7 & .244 & 2.037 & 94.148 & & & & & & \\
\hline 8 & .210 & 1.751 & 95.899 & & & & & & \\
\hline 9 & .153 & 1.272 & 97.171 & & & & & & \\
\hline 10 & .136 & 1.136 & 98.307 & & & & & & \\
\hline 11 & .109 & .911 & 99.218 & & & & & & \\
\hline 12 & .094 & .782 & 100.000 & & & & & & \\
\hline
\end{tabular}

Extraction Method: Principal Component Analysis. 
Table 9. Rotated Component Matrix ${ }^{\mathrm{a}}$ - Independent Variables

\begin{tabular}{|l|r|r|r|r|}
\hline & \multicolumn{5}{|c|}{ Component } \\
\cline { 2 - 5 } & 1 & 2 & 3 & 4 \\
\hline PREMI2 & .840 & & & \\
PREMI3 & .762 & & & \\
PREMI1 & .702 & & & \\
PREMI4 & .696 & & & \\
INVES2 & & .793 & & \\
RISK4 & & .761 & & \\
INVES4 & & .706 & & \\
INFO3 & & & .768 & \\
INFO1 & & & .720 & \\
INFO2 & & & .537 & \\
RISK2 & & & & .794 \\
RISK1 & & & & \\
\hline
\end{tabular}

Extraction Method: Principal Component Analysis.

Rotation Method: Varimax with Kaiser Normalization.

a. Rotation converged in 7 iterations.

Table 10. KMO and Bartlett's Test - Independent Variable

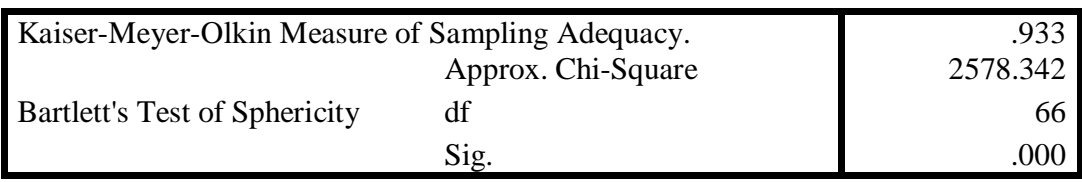

Table 11. Communalities - Independent Variables

\begin{tabular}{|l|r|r|}
\hline & \multicolumn{1}{|c|}{ Initial } & Extraction \\
\hline INFO1 & 1.000 & .885 \\
INFO2 & 1.000 & .786 \\
INFO3 & 1.000 & .902 \\
PREMI1 & 1.000 & .872 \\
PREMI2 & 1.000 & .907 \\
PREMI3 & 1.000 & .847 \\
INVES2 & 1.000 & .901 \\
INVES4 & 1.000 & .877 \\
RISK2 & 1.000 & .881 \\
RISK4 & 1.000 & .834 \\
PREMI4 & 1.000 & .857 \\
RISK1 & 1.000 & .848 \\
\hline
\end{tabular}

Extraction Method: Principal Component Analysis. 
Table 12. Total Variance Explained - Independent Variables

\begin{tabular}{|c|c|c|c|c|c|c|c|c|c|}
\hline \multirow[t]{2}{*}{ Component } & \multicolumn{3}{|c|}{ Initial Eigenvalues } & \multicolumn{3}{|c|}{$\begin{array}{c}\text { Extraction Sums of Squared } \\
\text { Loadings }\end{array}$} & \multicolumn{3}{|c|}{$\begin{array}{c}\text { Rotation Sums of Squared } \\
\text { Loadings } \\
\end{array}$} \\
\hline & Total & $\begin{array}{c}\% \text { of } \\
\text { Variance }\end{array}$ & $\begin{array}{c}\text { Cumulative } \\
\%\end{array}$ & Total & $\begin{array}{c}\% \text { of } \\
\text { Variance }\end{array}$ & $\begin{array}{c}\text { Cumulative } \\
\%\end{array}$ & Total & $\begin{array}{c}\% \text { of } \\
\text { Variance }\end{array}$ & $\begin{array}{c}\text { Cumulative } \\
\%\end{array}$ \\
\hline 1 & 8.708 & 72.569 & 72.569 & 8.708 & 72.569 & 72.569 & 3.246 & 27.053 & 27.053 \\
\hline 2 & .726 & 6.051 & 78.620 & .726 & 6.051 & 78.620 & 2.846 & 23.720 & 50.774 \\
\hline 3 & .518 & 4.317 & 82.937 & .518 & 4.317 & 82.937 & 2.289 & 19.076 & 69.850 \\
\hline 4 & .444 & 3.699 & 86.637 & .444 & 3.699 & 86.637 & 2.014 & 16.787 & 86.637 \\
\hline 5 & .354 & 2.949 & 89.586 & & & & & & \\
\hline 6 & .303 & 2.525 & 92.111 & & & & & & \\
\hline 7 & .244 & 2.037 & 94.148 & & & & & & \\
\hline 8 & .210 & 1.751 & 95.899 & & & & & & \\
\hline 9 & .153 & 1.272 & 97.171 & & & & & & \\
\hline 10 & .136 & 1.136 & 98.307 & & & & & & \\
\hline 11 & .109 & .911 & 99.218 & & & & & & \\
\hline 12 & .094 & .782 & 100.000 & & & & & & \\
\hline
\end{tabular}

Extraction Method: Principal Component Analysis.

Table 13. Rotated Component Matrix ${ }^{\mathrm{a}}$ - Independent Variables

\begin{tabular}{|l|r|r|r|r|}
\hline & \multicolumn{5}{|c|}{ Component } \\
\cline { 2 - 5 } & \multicolumn{1}{|c|}{1} & 2 & 3 & 4 \\
\hline PREMI2 & .840 & & & \\
PREMI3 & .762 & & & \\
PREMI1 & .702 & & & \\
PREMI4 & .696 & & & \\
INVES2 & & .793 & & \\
RISK4 & & .761 & & \\
INVES4 & & .706 & & \\
INFO3 & & & .768 & \\
INFO1 & & & .720 & \\
INFO2 & & & .537 & \\
RISK2 & & & & .794 \\
RISK1 & & & & \\
\hline
\end{tabular}

Extraction Method: Principal Component Analysis.

Rotation Method: Varimax with Kaiser Normalization.

a. Rotation converged in 7 iterations.

\section{B. Reliability Test}

Reliability test were conducted to find out the cronbach's alpha value which must be greater 0.6 with each variable checked one by one. Dependent variables have qualified of Cronbach's Alpha with .920 followed by independent variable passed the reliability test by INFO (.909), PREMI (.936), INVES (.925), and RISK (.858).

Table 14. Reliability Statistics - Dependent Variable

\begin{tabular}{|c|c|}
\hline Cronbach's Alpha & N of Items \\
\hline .920 & 4 \\
\hline
\end{tabular}

Table 15. Reliability Statistics - Independent Variable 
Reliability Statistics

\begin{tabular}{|r|r|}
\hline Cronbach's Alpha & N of Items \\
\hline .909 & 3 \\
\hline
\end{tabular}

PREMIUM

\section{Reliability Statistics}

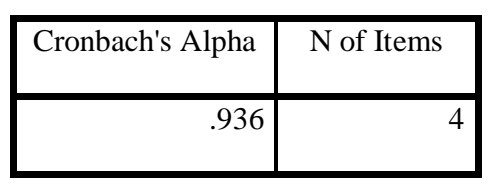

INVES (INVES1 \& INVES3 DELETED)

Reliability Statistics

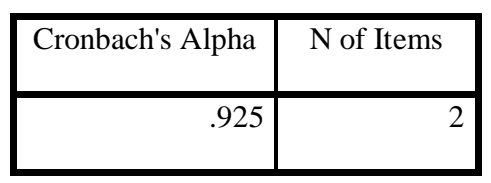

\section{RISK (RISK3 DELETED)}

\section{Reliability Statistics}

\begin{tabular}{|r|r|}
\hline Cronbach's Alpha & N of Items \\
\hline .858 & 3 \\
\hline
\end{tabular}

\section{Respondent's Profile}

Total respondents of this research were 200 respondents with six categories to get demographical respondents; age, education, occupation, income, and expense. Following figures summarized the results.

1. From $100 \%$, there were $51 \%$ female and $49 \%$ male.

2. Majority of respondents were between 25-30 years old with 59\%, 31-40 years old were 29\%, 41-50 years old were $11 \%$, and above 50 years old were only $1 \%$.

3. $66 \%$ respondents are bachelor degree graduates, $19 \%$ are post-graduate degree, $14 \%$ of respondents are senior high school graduates as the latest education, and $1 \%$ of respondents as others.

4. $60 \%$ of respondents' works in private company, 23 of respondents are entrepreneurs, $11 \%$ of respondents work as civil servant (work in government institution) and others were $6 \%$.

5. $41 \%$ respondents have income range between Rp. 5,000,001 - Rp. 10,000,000. 19\% of respondents have income between Rp. 3,000,000 - Rp. 5,000,000. 19\% of respondents have income between Rp. 10,000,001 - Rp. 15,000,000. 14\% respondents have income below Rp. 3,000,000 and 11\% above Rp. $15,000,000$.

6. $37 \%$ of respondents spend between Rp.3,000,000 - Rp.5,000,000 per month, $29 \%$ spend between Rp.5,000,001 - Rp.10,000,000 per month, 14\% spend below RP.3,000,000 per month, 9\% spend between Rp.10,000,001 - Rp.15,000,000 per month, and 11\% spend above Rp.15,000,000 per month. 


\section{Descriptive analysis}

Table 16. Information Data Summary

\begin{tabular}{|c|c|c|c|c|c|c|c|}
\hline \multirow[b]{2}{*}{ Questions } & 1 & 2 & 3 & 4 & 5 & 6 & 7 \\
\hline & $\begin{array}{l}\mathbf{F} \\
\%\end{array}$ & $\begin{array}{l}\mathbf{F} \\
\%\end{array}$ & $\begin{array}{l}\mathbf{F} \\
\%\end{array}$ & $\begin{array}{l}\mathbf{F} \\
\%\end{array}$ & $\begin{array}{l}\mathbf{F} \\
\%\end{array}$ & $\begin{array}{l}\mathbf{F} \\
\%\end{array}$ & $\begin{array}{l}\mathbf{F} \\
\%\end{array}$ \\
\hline $\begin{array}{l}\text { INFO1. Prudential provides me with } \\
\text { detailed information about the Unit Link } \\
\text { product }\end{array}$ & $\begin{array}{c}4 \\
2 \%\end{array}$ & $\begin{array}{r}10 \\
5 \%\end{array}$ & $\begin{array}{c}20 \\
10 \%\end{array}$ & $\begin{array}{c}20 \\
10 \%\end{array}$ & $\begin{array}{c}68 \\
34 \%\end{array}$ & $\begin{array}{c}56 \\
28 \%\end{array}$ & $\begin{array}{c}22 \\
11 \%\end{array}$ \\
\hline $\begin{array}{l}\text { INFO2. Prudential has made available a lot } \\
\text { of adequate coverage that meet my needs }\end{array}$ & $\begin{array}{c}4 \\
2 \%\end{array}$ & $\begin{array}{c}6 \\
3 \%\end{array}$ & $\begin{array}{c}26 \\
13 \%\end{array}$ & $\begin{array}{c}24 \\
12 \%\end{array}$ & $\begin{array}{c}64 \\
32 \%\end{array}$ & $\begin{array}{c}46 \\
23 \%\end{array}$ & $\begin{array}{c}30 \\
15 \%\end{array}$ \\
\hline $\begin{array}{l}\text { INFO3. Prudential give me sufficient } \\
\text { advance information regarding policy } \\
\text { renewal }\end{array}$ & $\begin{array}{c}2 \\
1 \%\end{array}$ & $\begin{array}{c}4 \\
2 \%\end{array}$ & $\begin{array}{c}22 \\
11 \%\end{array}$ & $\begin{array}{c}34 \\
17 \%\end{array}$ & $\begin{array}{c}52 \\
26 \%\end{array}$ & $\begin{array}{c}50 \\
25 \%\end{array}$ & $\begin{array}{c}36 \\
18 \%\end{array}$ \\
\hline $\begin{array}{l}\text { INFO4. I always easy to get information } \\
\text { about Unit Link policies through Prudential } \\
\text { Agents }\end{array}$ & $\begin{array}{c}2 \\
1 \%\end{array}$ & $\begin{array}{c}8 \\
4 \%\end{array}$ & $\begin{array}{r}16 \\
8 \%\end{array}$ & $\begin{array}{c}22 \\
11 \%\end{array}$ & $\begin{array}{c}70 \\
35 \%\end{array}$ & $\begin{array}{c}50 \\
25 \%\end{array}$ & $\begin{array}{c}32 \\
16 \%\end{array}$ \\
\hline
\end{tabular}

Source: Developed by Author

Table 16 shows summary from respondents from scale of 1 to 7 regarding the way customer get th information about Prulink. 34\% respondents somewhat agree the Prudential provides detail information about Prulink and $32 \%$ as adequate coverage said that the given information fit to their needs. In terms of delivering information regarding renewal, 26\% respondents answered that the agent has done a good job, since 35\% respondents somewhat agree that it is easy to get the information from agents.

Table 17. Premium Data Summary

\begin{tabular}{|c|c|c|c|c|c|c|c|}
\hline & 1 & 2 & 3 & 4 & 5 & 6 & 7 \\
\hline Questions & $\begin{array}{l}\mathbf{F} \\
\%\end{array}$ & $\begin{array}{l}\mathbf{F} \\
\%\end{array}$ & $\begin{array}{l}\mathbf{F} \\
\%\end{array}$ & $\begin{array}{l}\mathbf{F} \\
\%\end{array}$ & $\begin{array}{l}\mathbf{F} \\
\%\end{array}$ & $\begin{array}{l}\mathbf{F} \\
\%\end{array}$ & $\begin{array}{l}\mathbf{F} \\
\%\end{array}$ \\
\hline $\begin{array}{l}\text { PREMI1. Premium charged by Prudential } \\
\text { Unit Link product is reasonable }\end{array}$ & $\begin{array}{c}4 \\
2 \%\end{array}$ & $\begin{array}{c}2 \\
1 \%\end{array}$ & $\begin{array}{l}12 \\
6 \%\end{array}$ & $\begin{array}{c}32 \\
16 \%\end{array}$ & $\begin{array}{c}68 \\
34 \%\end{array}$ & $\begin{array}{c}50 \\
25 \%\end{array}$ & $\begin{array}{c}32 \\
16 \%\end{array}$ \\
\hline $\begin{array}{l}\text { PREMI2. Unit Link product is suitable for my } \\
\text { budget }\end{array}$ & $\begin{array}{c}4 \\
2 \%\end{array}$ & $\begin{array}{c}6 \\
3 \%\end{array}$ & $\begin{array}{c}8 \\
4 \%\end{array}$ & $\begin{array}{c}34 \\
17 \%\end{array}$ & $\begin{array}{c}58 \\
29 \%\end{array}$ & $\begin{array}{c}56 \\
28 \%\end{array}$ & $\begin{array}{c}34 \\
17 \%\end{array}$ \\
\hline $\begin{array}{l}\text { PREMI3. Prudential makes me comfortable } \\
\text { by offering different ways of payment }\end{array}$ & $\begin{array}{c}2 \\
1 \%\end{array}$ & $\begin{array}{c}8 \\
4 \%\end{array}$ & $\begin{array}{l}10 \\
5 \%\end{array}$ & $\begin{array}{c}26 \\
13 \%\end{array}$ & $\begin{array}{c}60 \\
30 \%\end{array}$ & $\begin{array}{c}64 \\
32 \%\end{array}$ & $\begin{array}{c}30 \\
15 \%\end{array}$ \\
\hline $\begin{array}{l}\text { PREMI4. Prudential gives me a flexible time } \\
\text { to pay the premium (determine by myself) }\end{array}$ & $\begin{array}{c}2 \\
1 \%\end{array}$ & $\begin{array}{c}6 \\
3 \%\end{array}$ & $\begin{array}{l}18 \\
9 \%\end{array}$ & $\begin{array}{c}26 \\
13 \%\end{array}$ & $\begin{array}{c}56 \\
28 \%\end{array}$ & $\begin{array}{c}58 \\
29 \%\end{array}$ & $\begin{array}{c}34 \\
17 \%\end{array}$ \\
\hline
\end{tabular}

Source: Developed by Author

Table 17 shows that the $34 \%$ respondents somewhat agree that the premium is reasonable to be paid. $29 \%$ said it is suitable to their budget and $32 \%$ also agree that Prudential that different way of payment give convenience to customers in paying the premium. $29 \%$ agree that there is flexibility to determine the payment due time. 
Table 18. Investment data summary

\begin{tabular}{|c|c|c|c|c|c|c|c|}
\hline & 1 & 2 & 3 & 4 & 5 & 6 & 7 \\
\hline Questions & $\begin{array}{l}\mathbf{F} \\
\%\end{array}$ & $\begin{array}{l}\mathbf{F} \\
\%\end{array}$ & $\begin{array}{l}\mathbf{F} \\
\%\end{array}$ & $\begin{array}{l}\mathbf{F} \\
\%\end{array}$ & $\begin{array}{l}\mathbf{F} \\
\%\end{array}$ & $\begin{array}{l}\mathbf{F} \\
\%\end{array}$ & $\begin{array}{l}\mathbf{F} \\
\%\end{array}$ \\
\hline $\begin{array}{l}\text { INVES1. Prudential Unit Link is a worth } \\
\text { investment }\end{array}$ & $\begin{array}{c}0 \\
0 \%\end{array}$ & $\begin{array}{l}12 \\
6 \%\end{array}$ & $\begin{array}{l}12 \\
6 \%\end{array}$ & $\begin{array}{c}26 \\
13 \%\end{array}$ & $\begin{array}{c}52 \\
26 \%\end{array}$ & $\begin{array}{c}58 \\
29 \%\end{array}$ & $\begin{array}{c}40 \\
20 \%\end{array}$ \\
\hline $\begin{array}{l}\text { INVES2. Prudential Unit Link has less risk in } \\
\text { comparison others }\end{array}$ & $\begin{array}{c}0 \\
0 \%\end{array}$ & $\begin{array}{c}14 \\
7 \%\end{array}$ & $\begin{array}{l}10 \\
5 \%\end{array}$ & $\begin{array}{c}32 \\
16 \%\end{array}$ & $\begin{array}{c}68 \\
34 \%\end{array}$ & $\begin{array}{c}48 \\
24 \%\end{array}$ & $\begin{array}{c}28 \\
14 \%\end{array}$ \\
\hline $\begin{array}{l}\text { INVES3. I am satisfied of the return on } \\
\text { investment offered by Prudential Unit link }\end{array}$ & $\begin{array}{c}0 \\
0 \%\end{array}$ & $\begin{array}{c}14 \\
7 \%\end{array}$ & $\begin{array}{l}16 \\
8 \%\end{array}$ & $\begin{array}{c}18 \\
9 \%\end{array}$ & $\begin{array}{c}76 \\
38 \%\end{array}$ & $\begin{array}{c}42 \\
21 \%\end{array}$ & $\begin{array}{c}34 \\
17 \%\end{array}$ \\
\hline $\begin{array}{l}\text { INVES4. I am satisfied with the schemes } \\
\text { offered by Prudential Unit Link }\end{array}$ & $\begin{array}{c}2 \\
1 \%\end{array}$ & 10 & $\begin{array}{l}16 \\
8 \%\end{array}$ & $\begin{array}{c}28 \\
14 \%\end{array}$ & $\begin{array}{c}54 \\
27 \%\end{array}$ & $\begin{array}{c}62 \\
31 \%\end{array}$ & $\begin{array}{c}28 \\
24 \%\end{array}$ \\
\hline
\end{tabular}

Source: Developed by Author

Table 18 explain 34\% respondents somewhat agree Prulink has less risk compare to other insurance product and $38 \%$ quite satisfy about the return on investment. 29\% agree that Prulink is worth the investment as well as the schemes offered $31 \%$.

Table 19. Risk Data Summary

\begin{tabular}{|c|c|c|c|c|c|c|c|}
\hline & 1 & 2 & 3 & 4 & 5 & 6 & 7 \\
\hline Questions & $\begin{array}{l}\mathbf{F} \\
\%\end{array}$ & $\begin{array}{l}\mathbf{F} \\
\%\end{array}$ & $\begin{array}{l}\mathbf{F} \\
\%\end{array}$ & $\begin{array}{l}\mathbf{F} \\
\%\end{array}$ & $\begin{array}{l}\mathbf{F} \\
\%\end{array}$ & $\begin{array}{l}\mathbf{F} \\
\%\end{array}$ & $\begin{array}{l}\mathbf{F} \\
\%\end{array}$ \\
\hline $\begin{array}{l}\text { RISK1. I buy a Unit Link product to face } \\
\text { unexpected situation in the future }\end{array}$ & $\begin{array}{c}2 \\
1 \%\end{array}$ & $\begin{array}{c}0 \\
0 \%\end{array}$ & $\begin{array}{c}24 \\
12 \%\end{array}$ & $\begin{array}{l}16 \\
8 \%\end{array}$ & $\begin{array}{c}72 \\
36 \%\end{array}$ & $\begin{array}{c}48 \\
24 \%\end{array}$ & $\begin{array}{c}38 \\
19 \%\end{array}$ \\
\hline $\begin{array}{l}\text { RISK2. My current job is susceptible risky, } \\
\text { So I choose to buy insurance }\end{array}$ & $\begin{array}{c}6 \\
3 \%\end{array}$ & $\begin{array}{c}8 \\
8 \%\end{array}$ & $\begin{array}{c}20 \\
10 \%\end{array}$ & $\begin{array}{c}42 \\
21 \%\end{array}$ & $\begin{array}{c}50 \\
\mathbf{2 5 \%}\end{array}$ & $\begin{array}{c}44 \\
22 \%\end{array}$ & $\begin{array}{c}30 \\
15 \%\end{array}$ \\
\hline $\begin{array}{l}\text { RISK3. Unit Link policy can cover the risk } \\
\text { of major loss }\end{array}$ & $\begin{array}{c}4 \\
2 \%\end{array}$ & $\begin{array}{c}4 \\
2 \%\end{array}$ & $\begin{array}{c}22 \\
11 \%\end{array}$ & $\begin{array}{c}32 \\
16 \%\end{array}$ & $\begin{array}{c}50 \\
25 \%\end{array}$ & $\begin{array}{c}50 \\
25 \%\end{array}$ & $\begin{array}{c}38 \\
19 \%\end{array}$ \\
\hline $\begin{array}{l}\text { RISK4. I buy a Unit Link product because } \\
\text { there is no risk of losing money that I paid }\end{array}$ & $\begin{array}{c}4 \\
2 \%\end{array}$ & $\begin{array}{l}12 \\
6 \%\end{array}$ & $\begin{array}{c}8 \\
4 \%\end{array}$ & $\begin{array}{c}40 \\
20 \%\end{array}$ & $\begin{array}{c}50 \\
25 \%\end{array}$ & $\begin{array}{c}60 \\
30 \%\end{array}$ & $\begin{array}{c}26 \\
13 \%\end{array}$ \\
\hline
\end{tabular}

Source: Developed by Author

Table 19 shows that $36 \%$ of total respondents somewhat agree that decision to purchase Prulink is to face unexpected risk and cover $25 \%$ said that to cover them in susceptible risk job. $25 \%$ respondents agree that Prulink can cover a major loss and $30 \%$ said there is no risk of losing the paid money.

Table 20. Purchase Decision Data Summary

\begin{tabular}{|c|c|c|c|c|c|c|c|}
\hline & 1 & 2 & 3 & 4 & 5 & 6 & 7 \\
\hline Questions & $\begin{array}{l}\mathbf{F} \\
\%\end{array}$ & $\begin{array}{l}\mathbf{F} \\
\%\end{array}$ & $\begin{array}{l}\mathbf{F} \\
\%\end{array}$ & $\begin{array}{l}\mathbf{F} \\
\%\end{array}$ & $\begin{array}{l}\mathbf{F} \\
\%\end{array}$ & $\begin{array}{l}\mathbf{F} \\
\%\end{array}$ & $\begin{array}{l}\mathbf{F} \\
\%\end{array}$ \\
\hline $\begin{array}{l}\text { PRDC1. Before I decide to purchase } \\
\text { Prudential Unit Link, I have to look for } \\
\text { product information actively }\end{array}$ & $\begin{array}{c}0 \\
0 \%\end{array}$ & $\begin{array}{c}4 \\
8 \%\end{array}$ & $\begin{array}{l}16 \\
8 \%\end{array}$ & $\begin{array}{c}18 \\
9 \%\end{array}$ & $\begin{array}{c}58 \\
29 \%\end{array}$ & $\begin{array}{c}60 \\
30 \%\end{array}$ & $\begin{array}{c}44 \\
22 \%\end{array}$ \\
\hline
\end{tabular}




\begin{tabular}{|c|c|c|c|c|c|c|c|}
\hline $\begin{array}{l}\text { PRDC2. After I get the information, I have to } \\
\text { evaluate and consider carefully before making } \\
\text { decisions }\end{array}$ & $\begin{array}{c}0 \\
0 \%\end{array}$ & $\begin{array}{c}4 \\
2 \%\end{array}$ & $\begin{array}{c}18 \\
9 \%\end{array}$ & $\begin{array}{c}20 \\
10 \%\end{array}$ & $\begin{array}{c}54 \\
27 \%\end{array}$ & $\begin{array}{c}54 \\
27 \%\end{array}$ & $\begin{array}{c}50 \\
25 \%\end{array}$ \\
\hline $\begin{array}{l}\text { PRDC3. I think to purchase Prudential Unit } \\
\text { Link is the right decision }\end{array}$ & $\begin{array}{c}0 \\
0 \%\end{array}$ & $\begin{array}{c}6 \\
3 \%\end{array}$ & $\begin{array}{c}18 \\
9 \%\end{array}$ & $\begin{array}{c}20 \\
10 \%\end{array}$ & $\begin{array}{c}54 \\
27 \%\end{array}$ & $\begin{array}{c}64 \\
32 \%\end{array}$ & $\begin{array}{c}38 \\
19 \%\end{array}$ \\
\hline $\begin{array}{l}\text { PRDC4. I am satisfied with the benefit offered } \\
\text { because it fulfill the needs of guarantee life } \\
\text { and health }\end{array}$ & $\begin{array}{c}0 \\
0 \%\end{array}$ & $\begin{array}{c}8 \\
4 \%\end{array}$ & $\begin{array}{l}12 \\
6 \%\end{array}$ & $\begin{array}{c}32 \\
16 \%\end{array}$ & $\begin{array}{c}60 \\
30 \%\end{array}$ & $\begin{array}{c}48 \\
24 \%\end{array}$ & $\begin{array}{c}40 \\
20 \%\end{array}$ \\
\hline
\end{tabular}

Table 20 shows that $30 \%$ respondents look for the information before make a decision while $27 \%$ respondents agree to evaluate the product before make a decision. $32 \%$ respondents agree that Prulink is the right decision and $30 \%$ respondents feel satisfied with the benefits because it fulfills their needs.

\section{E. Data Analysis}

This research used SPSS ver .20 to do multiple regression analysis. There are two kind of analysis resulted; classical assumption test analysis and hypothesis test analysis. Classical assumption explained by figure 2 for histogram and Figure 3 for Normal P-P Plot for the normality that conclude the assumption of normality is normal.

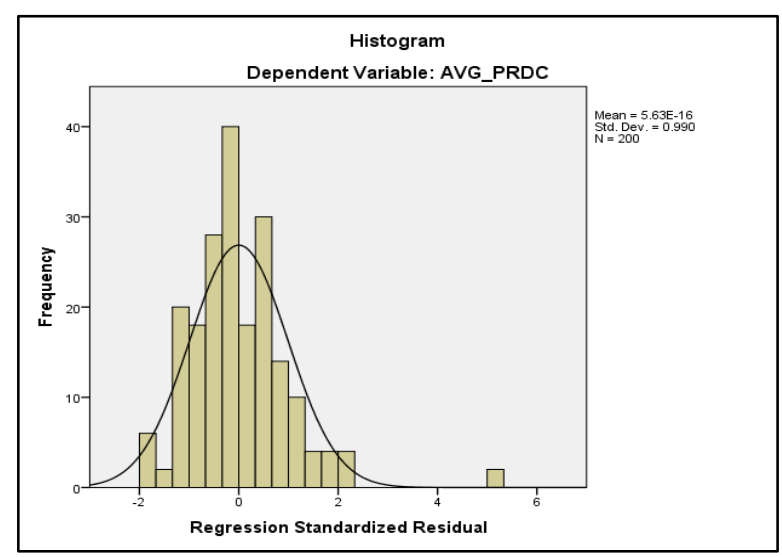

Figure 2. Histogram

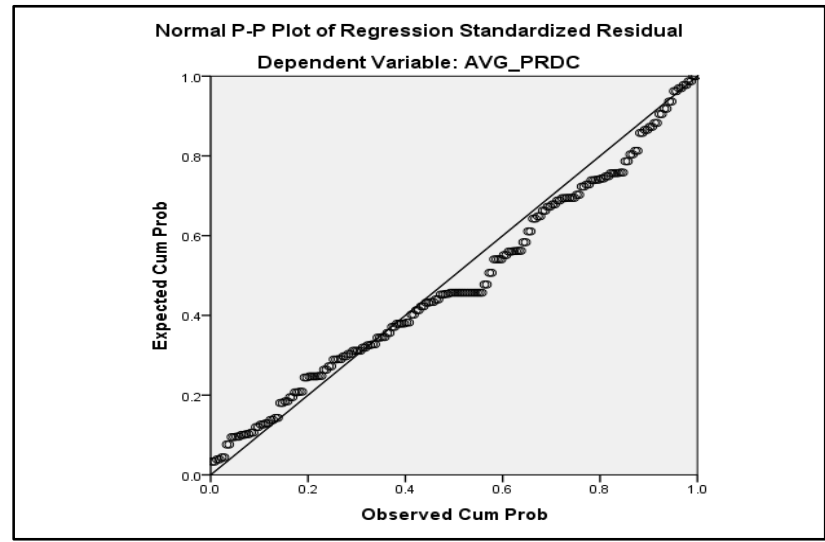

Figure 3. Normal P-P Plot 
Figure 4 explained that the dots in this research are spread randomly without creating certain systemic pattern such as increasing or decreasing pattern. From that result, it can be concluded that there is no heteroscedascity in this research.

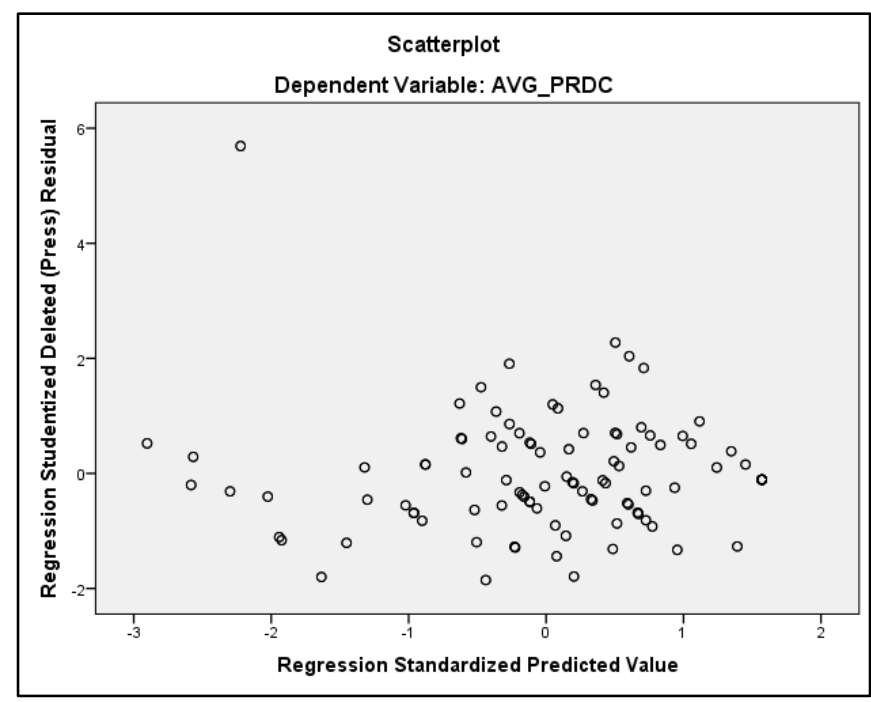

Figure 4. Heteroscedascity

In order to test whether the regression model found a correlation between the independent variables, multicollinearity test were conducted. Table 21 shows that variance inflation factor greater than 0,1 but less than 10 and the number of tolerance is less than 1. From that result, it concluded that there is no multicollinearity.

Table 21. Multicollinearity

\section{Coefficients $^{\mathrm{a}}$}

\begin{tabular}{|c|c|c|c|c|c|c|c|c|}
\hline \multirow{2}{*}{\multicolumn{2}{|c|}{ Model }} & \multicolumn{2}{|c|}{ Unstandardized Coefficients } & \multirow{2}{*}{$\begin{array}{c}\text { Standardized } \\
\text { Coefficients } \\
\text { Beta }\end{array}$} & \multirow[t]{2}{*}{$\mathrm{t}$} & \multirow[t]{2}{*}{ Sig. } & \multicolumn{2}{|c|}{ Collinearity Statistics } \\
\hline & & B & Std. Error & & & & Tolerance & VIF \\
\hline \multirow{5}{*}{1} & (Constant) & .710 & .156 & & 4.544 & .000 & & \\
\hline & AVG_INFO & .192 & .057 & .214 & 3.352 & .001 & .213 & 4.686 \\
\hline & AVG_PREMI & .479 & .053 & .501 & 8.956 & .000 & .278 & 3.603 \\
\hline & AVG_INVES & .159 & .054 & .180 & 2.962 & .003 & .234 & 4.270 \\
\hline & AVG_RISK & .075 & .061 & .080 & 1.241 & .216 & .207 & 4.835 \\
\hline
\end{tabular}

a. Dependent Variable: AVG_PRDC

Table 22. F- Test

ANOVA $^{a}$

\begin{tabular}{|ll|r|r|r|r|r|}
\hline Model & Sum of Squares & df & Mean Square & F & Sig. \\
\hline \multirow{3}{*}{1} & Regression & 229.219 & 4 & 57.305 & 239.566 & $.000^{\mathrm{b}}$ \\
& Residual & 46.644 & 195 & .239 & & \\
& Total & 275.864 & 199 & & & \\
\hline
\end{tabular}

a. Dependent Variable: AVG_PRDC

b. Predictors: (Constant), AVG_RISK, AVG_PREMI, AVG_INVES, AVG_INFO 
Based on table 22, it shown that sig. value is .000 lower than 0.05 means that there is a significant influence of customer perception aspects as independent variables on purchase decision as dependent variable altogether. In order to check whether independent variable is strong predictor of dependent variable, sig. value on each independent variable shown in table 8 should be lower than 0.05 . The result found that all independent variables influence purchase decision because it exposes partially.

Table 23. Adjusted Coefficient Determination $\left(\mathrm{R}^{2}\right)$

Model Summary ${ }^{\mathrm{b}}$

\begin{tabular}{l|r|r|r|r|r|}
\hline Model & R & R Square & Adjusted R Square & $\begin{array}{c}\text { Std. Error of the } \\
\text { Estimate }\end{array}$ & Durbin-Watson \\
\hline 1 & $.912^{\mathrm{a}}$ & .831 & & .827 & .48908 \\
\hline
\end{tabular}
a. Predictors: (Constant), AVG_RISK, AVG_PREMI, AVG_INVES, AVG_INFO
b. Dependent Variable: AVG_PRDC

Table 23 shows that the number of adjusted $\mathrm{R}$ square $\left(\mathrm{R}^{2}\right)$ of independent variables is .827 influence dependent variable meaning that purchase decision is influenced by customer perception by $82.7 \%$ while the rest are influenced by other variable that beyond of this research.

\section{F. Interpretation of Result}

Based on the data analysis, information has influence on purchase decision, and has similar result with past research of Ubinaite et.al, (2013) which stated that information has high influence on future purchase. Premium has significant influence on purchase decision. Sebjan \& Tomnic (2015), found that premium is the most significant factor among the customer perception, thus the result of premium supports the previous research. Influence has influence on purchase decision, considering the case is focus on unit link insurance product. Bhatnagar (2015), said that customers not only buy unit link insurance product to get protection but also to have role to become investor. Result of T-test shows that risk has no significant influences on purchase decision and contrast with theory by Redja (2011) that said insurance exist to transfer the risk. Meanwhile, this research found that customer perception about risk is not really influence the decision-making. Research of Campbell Institute (2014) about risk perception said that a person who has lower risk perception tend to has higher risk tolerance. Based on that statement from Campbell institute, it is found that the reason why risk has no significant influence to purchase decision that because customers think that unit link insurance product has value added in terms of investment instead only protection.

Based on the F-test result that all the aspect of customer perception has significant influence on purchase decision is supported by Reddy \& Jahangir, (2015) who said that consumer behavior is caused by the perception on each individual. Perception is a mind that affects to act and decide. Even though, there is no partial significant influence from risk to purchase decision, as overall the four aspects as influenced customer to make purchase decision on buying Prulink.

Varghese (2013) stated in his study that there are marketing factor and social factor that take part in creating strong influence instead of personal factor. Marketing role can be performed through insurance agent who presents the product with protection and investment plan directly to customer. Good agent must make customers aware and attracted to the product. Unique and eye catching advertisement in social media also attempt to attract potential customer. As for social factor, neighborhood and experience from inner cycle might influence purchase decision. Those marketing and social role reveals the other $17.3 \%$ of other factors as a result of Adjusted R square $\left(\mathrm{R}^{2}\right)$ on table 14 .

\section{CONCLUSION}

Based on the hypothesis testing through multiple regression analysis, some results conclude to achieve research objective:

1. This research found that information factor has significant partial influence on purchase decision. 
2. This research found that premium factor has significant partial influence on purchase decision.

3. This research found that investment factor has significant partial influence on purchase decision.

4. This research found that risk factor has no significant partial influence on purchase decision.

5. This research found that customer perception has significant influence on purchase decision.

\section{A. Managerial Implications}

PT. Prudential Life Assurance is not sole player in unit link insurance product in Indonesia. There are many unit link insurance products offered by another insurance company such as AIA, Manulife Financial, Asuransi Cigna Indonesia and etc. This research can be utilized to evaluate company performance given the situation that each unit link insurance product provider has different way in offering their product to customer. Based on the result and discussion, company need to maintain customer loyalty by consistently update the information about Prulink product.

\section{B. Future Research}

Since this research only focus on unit link insurance product from Prudential (Prulink) without considering competition between unit link insurance provider, it is possible that future research utilize same variables with broader target not only to prudential customers but also unit link insurance product customers from competitors and also with broader coverage not only in Jakarta but also other big cities in Indonesia. The difference between agent in offering insurance product also can be evaluated for future research since every unit link insurance product provide has different way in promoting and offering the product to the potential customer.

\section{REFERENCES}

Beritasatu.com. (2016, October 206). AAJI Perkirakan Premi Asuransi Jiwa Tumbuh 15\%. Retrieved January 01, 2017, from: http://www.beritasatu.com/asuransi/391808-aaji-perkirakan-premi-asuransi-jiwatumbuh-15.html

Bhatnagar, V. K. (2015). Factors Affecting Investors Perceptions' towards Investment in ULIP Doctoral dissertation). University of Rajasthan, Jaipur.

Bisnis.com. (2016, November 18). AAJI: Kuartal III/2016, Pendapatan Asuransi Jiwa Tumbuh 78,1\%. Retrieved January 01, 2017, from: http://finansial.bisnis.com/read/20161118/215/604100/aaji-kuartaliii2016-pendapatan-asuransi-jiwa-tumbuh-781

CampbellInstittute. (2014). Risk Perception: Theories, Strategies, and next steps. Retrieved March 17, 2017.

Ginting, E. A. (2016). The Influence of Income, Risk, Insurance, Premium and Information on The Purchasing

Decision of Health Insurance Prodcts (A Case study of PT. Prudential Life Assurance South Jakarta Area).

President Unviersity, Cikarang

Huitt, W. (2007). Maslow's Hierarchy of Needs. Educational Psychology Interactive. Valdosta, GA: Valdosta State University. $\quad$ Retrieved February $15, \quad$ 2017, from: http://www.edpsycinteractive.org/topics/regsys/maslow.html

Infobanknews.com. (2016, February 26). Premi Unit link Ditaksir Tumbuh 15\%. Retrieved January 01, 2017, from http://infobanknews.com/premi-unit-link-ditaksir-tumbuh-15/

Lovelock, C., \& Wirtz, J. (2007). Services Marketing. New Jersey: Pearson Prentice Hall.

Malhotra, N. K. (2009). Basic Marketing Research - A Decision Making Aprroach, third Edition. New Jersey: Pearson International Global.

Redja, G. E. (2011). Principles of Risk Management and Insurance. Boston: Pearson.

Robbins, S. P., \& Judge, T. A. (2011). Organizational Behaviour, Fourtheen Edition. USA: Pearson International Edition. 
Rajeswari, K., \& Kartheeswari, S. (2011). Perception of Customers Towards Life Insurance Services. Asia Pacific Journal of Research in Business Management, 2(12).

Reddy, P. R., \& Jahangir, Y. (2015). Consumer Buying Behaviour towards Life Insurance Products in Rural Market 'A Case Study of Nalgonda District, Telangana State. International Journal of Scientific Research, 4(3).

Salim, L. I. P. (2012). Analisis Faktor-Faktor yang Mempengaruhi Keputusan Pembelian Konsumen Pada Produk Asuransi. Universitas Hasanuddin, Makassar.

Sebjan, U., \& Tominc, P. (2015). Conceptual Model of Relationships Among Customer Perceptions of Components of Insurance Service. South East European Journal of Economics and Business, 10(1).

Ulbinaite, A., Kucinskiene, M., \& Moullec, Y. L. (2014). The complexity of the insurance purchase decision making process. Transformations in Business and Economics, 13(3).

Varghese, T. (2013). Determinants of Consumer Purchase Decisions of Health Insurance in Kerala (Thesis Doctoral). Cochin University of Science and Technology, India. 